Bull. Korean Math. Soc. 48 (2011), No. 3, pp. 627-636

DOI 10.4134/BKMS.2011.48.3.627

\title{
SANDWICH THEOREMS FOR HIGHER-ORDER DERIVATIVES OF $p$-VALENT FUNCTIONS DEFINED BY CERTAIN LINEAR OPERATOR
}

\author{
Mohamed K. Aouf and Tamer M. Seoudy
}

\begin{abstract}
In this paper, we obtain some applications of first order differential subordination and superordination results for higher-order derivatives of $p$-valent functions involving certain linear operator. Some of our results improve and generalize previously known results.
\end{abstract}

\section{Introduction}

Let $H(U)$ be the class of analytic functions in the open unit disk $U=\{z \in$ $\mathbb{C}:|z|<1\}$ and let $H[a, p]$ be the subclass of $H(U)$ consisting of functions of the form:

$$
f(z)=a+a_{p} z^{p}+a_{p+1} z^{p+1}+\cdots(a \in \mathbb{C}) .
$$

For simplicity $H[a]=H[a, 1]$. Also, let $\mathcal{A}(p)$ be the subclass of $H(U)$ consisting of functions of the form:

$$
f(z)=z^{p}+\sum_{k=p+1}^{\infty} a_{k} z^{k} \quad(p \in \mathbb{N}=\{1,2, \ldots\}) .
$$

which are $p$-valent in $U$. If $f, g \in H(U)$, we say that $f$ is subordinate to $g$ or $f$ is superordinate to $g$, written $f(z) \prec g(z)$ if there exists a Schwarz function $\omega$, which (by definition) is analytic in $U$ with $\omega(0)=0$ and $|\omega(z)|<1$ for all $z \in U$, such that $f(z)=g(\omega(z)), z \in U$. Furthermore, if the function $g$ is univalent in $U$, then we have the following equivalence (cf., e.g., [6], [9] and $[10])$ :

$$
f(z) \prec g(z) \Leftrightarrow f(0)=g(0) \text { and } f(U) \subset g(U) .
$$

Let $\phi: \mathbb{C}^{2} \times U \rightarrow \mathbb{C}$ and $h(z)$ be univalent in $U$. If $p(z)$ is analytic in $U$ and satisfies the first order differential subordination:

$$
\phi\left(p(z), z p^{\prime}(z) ; z\right) \prec h(z),
$$

Received October 22, 2009.

2010 Mathematics Subject Classification. 30C45.

Key words and phrases. analytic function, Hadamard product, differential subordination, superordination, linear operator. 
then $p(z)$ is a solution of the differential subordination (1.3). The univalent function $q(z)$ is called a dominant of the solutions of the differential subordination (1.3) if $p(z) \prec q(z)$ for all $p(z)$ satisfying (1.3). A univalent dominant $\tilde{q}$ that satisfies $\tilde{q} \prec q$ for all dominants of (1.3) is called the best dominant. If $p(z)$ and $\phi\left(p(z), z p^{\prime}(z) ; z\right)$ are univalent in $U$ and if $p(z)$ satisfies first order differential superordination:

$$
h(z) \prec \phi\left(p(z), z p^{\prime}(z) ; z\right),
$$

then $p(z)$ is a solution of the differential superordination (1.4). An analytic function $q(z)$ is called a subordinant of the solutions of the differential superordination (1.4) if $q(z) \prec p(z)$ for all $p(z)$ satisfying (1.4). A univalent subordinant $\tilde{q}$ that satisfies $q \prec \tilde{q}$ for all subordinants of (1.4) is called the best subordinant. Using the results of Miller and Mocanu [10], Bulboaca [5] considered certain classes of first order differential superordinations as well as superordination-preserving integral operators [6]. Ali et al. [1], have used the results of Bulboaca [5] to obtain sufficient conditions for normalized analytic functions $f \in \mathcal{A}(1)$ to satisfy:

$$
q_{1}(z) \prec \frac{z f^{\prime}(z)}{f(z)} \prec q_{2}(z),
$$

where $q_{1}$ and $q_{2}$ are given univalent functions in $U$ with $q_{1}(0)=q_{2}(0)=1$. Also, Tuneski [15] obtained a sufficient condition for starlikeness of $f \in \mathcal{A}(1)$ in terms of the quantity $\frac{f^{\prime \prime}(z) f(z)}{\left(f^{\prime}(z)\right)^{2}}$. Recently, Shanmugam et al. [14] obtained sufficient conditions for the normalized analytic function $f \in \mathcal{A}(1)$ to satisfy

$$
q_{1}(z) \prec \frac{f(z)}{z f^{\prime}(z)} \prec q_{2}(z)
$$

and

$$
q_{1}(z) \prec \frac{z^{2} f^{\prime}(z)}{\{f(z)\}^{2}} \prec q_{2}(z) .
$$

They [14] also obtained results for functions defined by using Carlson-Shaffer operator [7], Ruscheweyh derivative [12] and Sălăgean operator [13].

Upon differentiating both sides of (1.1) $j$-times with respect and to $z$, we have

$$
f^{(j)}(z)=\delta(p ; j) z^{p-j}+\sum_{k=p+1}^{\infty} \delta(k ; j) a_{k} z^{k-j}
$$

where

$$
\delta(p ; j)=\frac{p !}{(p-j) !} \quad\left(p>j ; p \in \mathbb{N} ; j \in \mathbb{N}_{0}=\mathbb{N} \cup\{0\}\right) .
$$

For a function $f \in \mathcal{A}(p)$, we define the linear operator $D_{p}^{n}: \mathcal{A}(p) \rightarrow \mathcal{A}(p)$ by:

$$
D_{p}^{0} f^{(j)}(z)=f^{(j)}(z)
$$




$$
\begin{aligned}
D_{p}^{1} f^{(j)}(z) & =D\left(f^{(j)}(z)\right) \\
& =\delta(p ; j) z^{p-j}+\sum_{k=p+1}^{\infty} \delta(k ; j)\left(\frac{k-j}{p-j}\right) a_{k} z^{k-j}, \\
D_{p}^{2} f^{(j)}(z) & =D\left(D_{p}^{1} f^{(j)}(z)\right) \\
& =\delta(p ; j) z^{p-j}+\sum_{k=p+1}^{\infty} \delta(k ; j)\left(\frac{k-j}{p-j}\right)^{2} a_{k} z^{k-j},
\end{aligned}
$$

and (in general)

$$
\begin{aligned}
D_{p}^{n} f^{(j)}(z)= & D\left(D_{p}^{n-1} f^{(j)}(z)\right) \\
= & \delta(p ; j) z^{p-j}+\sum_{k=p+1}^{\infty} \delta(k ; j)\left(\frac{k-j}{p-j}\right)^{n} a_{k} z^{k-j} \\
& \left(p>j ; p, n \in \mathbb{N} ; j \in \mathbb{N}_{0} ; z \in U\right) .
\end{aligned}
$$

From (1.7), we can easily deduce that

$$
\text { (1.8) } z\left(D_{p}^{n} f^{(j)}(z)\right)^{\prime}=(p-j) D_{p}^{n+1} f^{(j)}(z) \quad\left(p>j ; p \in \mathbb{N} ; n, j \in \mathbb{N}_{0} ; z \in U\right) \text {. }
$$

The operator $D_{p}^{n} f^{(j)}(z) \quad\left(p>j, p \in \mathbb{N}, n, j \in \mathbb{N}_{0}\right)$ was introduced and studied by Aouf $[2,3]$ where

$$
f(z)=z^{p}-\sum_{k=p+1}^{\infty} a_{k} z^{k} \quad\left(a_{k} \geq 0\right)
$$

We note that

(i) the differential operator $D_{p}^{n} f^{(0)}(z)=D_{p}^{n} f(z)$ was introduced by Kamali and Orhan [8] and Aouf and Mostafa [4];

(ii) the differential operator $D_{1}^{n} f^{(0)}(z)=D^{n} f(z)$ was introduced by Sălăgean [13].

In this paper, we will derive several subordination results, superordination results and sandwich results involving the operator $D_{p}^{n} f^{(j)}(z)$.

\section{Definitions and preliminaries}

In order to prove our subordinations and superordinations, we need the following definition and lemmas.

Definition 1 ([10]). Denote by $Q$, the set of all functions $f$ that are analytic and injective on $\bar{U} \backslash E(f)$, where

$$
E(f)=\left\{\zeta \in \partial U: \lim _{z \rightarrow \zeta} f(z)=\infty\right\},
$$

and are such that $f^{\prime}(\zeta) \neq 0$ for $\zeta \in \partial U \backslash E(f)$. 
Lemma 1 ([10]). Let $q(z)$ be univalent in $U$ and $\theta$ and $\varphi$ be analytic in a domain $D$ containing $q(U)$ with $\varphi(w) \neq 0$ when $w \in q(U)$. Set

$$
\psi(z)=z q^{\prime}(z) \varphi(q(z)) \quad \text { and } \quad h(z)=\theta(q(z))+\psi(z) .
$$

\section{Suppose that}

(i) $\psi(z)$ is starlike univalent in $U$,

(ii) $\Re\left\{\frac{z h^{\prime}(z)}{\psi(z)}\right\}>0$ for $z \in U$.

If $p(z)$ is analytic with $p(0)=q(0), p(U) \subset D$ and

$$
\theta(p(z))+z p^{\prime}(z) \varphi(p(z)) \prec \theta(q(z))+z q^{\prime}(z) \varphi(q(z)),
$$

then $p(z) \prec q(z)$ and $q(z)$ is the best dominant.

Taking $\theta(w)=\alpha w$ and $\varphi(w)=\gamma$ in Lemma 1, Shanmugam et al. [14] obtained the following lemma.

Lemma 2 ([14]). Let $q(z)$ be univalent in $U$ with $q(0)=1$. Let $\alpha \in \mathbb{C}, \gamma \in \mathbb{C}^{*}$, further assume that

$$
\Re\left\{1+\frac{z q^{\prime \prime}(z)}{q^{\prime}(z)}\right\}>\max \left\{0,-\Re\left(\frac{\alpha}{\gamma}\right)\right\} .
$$

If $p(z)$ is analytic in $U$, and

$$
\alpha p(z)+\gamma z p^{\prime}(z) \prec \alpha q(z)+\gamma z q^{\prime}(z)
$$

then $p(z) \prec q(z)$ and $q(z)$ is the best dominant.

Lemma $3([5])$. Let $q(z)$ be convex univalent in $U$ and $\vartheta$ and $\phi$ be analytic in a domain $D$ containing $q(U)$. Suppose that

(i) $\Re\left\{\frac{\vartheta^{\prime}(q(z))}{\phi(q(z))}\right\}>0$ for $z \in U$,

(ii) $\Psi(z)=z q^{\prime}(z) \phi(q(z))$ is starlike univalent in $U$.

If $p(z) \in H[q(0), 1] \cap Q$, with $p(U) \subseteq D$, and $\vartheta(p(z))+z p^{\prime}(z) \phi(p(z))$ is univalent in $U$ and

$$
\vartheta(q(z))+z q^{\prime}(z) \phi(q(z)) \prec \vartheta(p(z))+z p^{\prime}(z) \phi(p(z)),
$$

then $q(z) \prec p(z)$ and $q(z)$ is the best subordinant.

Taking $\vartheta(w)=\alpha w$ and $\phi(w)=\gamma$ in Lemma 3, Shanmugam et al. [14] obtained the following lemma.

Lemma 4 ([14]). Let $q(z)$ be convex univalent in $U, q(0)=1$. Let $\alpha \in \mathbb{C}$, $\gamma \in \mathbb{C}^{*}$ and $\Re\left(\frac{\alpha}{\gamma}\right)>0$. If $p(z) \in H[q(0), 1] \cap Q, \alpha p(z)+\gamma z p^{\prime}(z)$ is univalent in $U$ and

$$
\alpha q(z)+\gamma z q^{\prime}(z) \prec \alpha p(z)+\gamma z p^{\prime}(z)
$$

then $q(z) \prec p(z)$ and $q(z)$ is the best subordinant. 


\section{Sandwich results}

Unless otherwise mentioned, we assume throughout this paper that $p>j$; $p \in \mathbb{N}$ and $n, j \in \mathbb{N}_{0}$.

Theorem 1. Let $q(z)$ be univalent in $U$ with $q(0)=1$, and $\gamma \in \mathbb{C}^{*}$. Further, assume that

$$
\Re\left\{1+\frac{z q^{\prime \prime}(z)}{q^{\prime}(z)}\right\}>\max \left\{0,-\Re\left(\frac{1}{\gamma}\right)\right\} .
$$

If $f \in \mathcal{A}(p)$ satisfy the following subordination condition:

$$
\frac{D_{p}^{n} f^{(j)}(z)}{D_{p}^{n+1} f^{(j)}(z)}+\gamma(p-j)\left\{1-\frac{D_{p}^{n} f^{(j)}(z) D_{p}^{n+2} f^{(j)}(z)}{\left[D_{p}^{n+1} f^{(j)}(z)\right]^{2}}\right\} \prec q(z)+\gamma z q^{\prime}(z)
$$

then

$$
\frac{D_{p}^{n} f^{(j)}(z)}{D_{p}^{n+1} f^{(j)}(z)} \prec q(z)
$$

and $q(z)$ is the best dominant.

Proof. Define a function $p(z)$ by

$$
p(z)=\frac{D_{p}^{n} f^{(j)}(z)}{D_{p}^{n+1} f^{(j)}(z)} \quad(z \in U) .
$$

Then the function $p(z)$ is analytic in $U$ and $p(0)=1$. Therefore, differentiating (3.3) logarithmically with respect to $z$ and using the identity (1.8) in the resulting equation, we have

$$
\frac{D_{p}^{n} f^{(j)}(z)}{D_{p}^{n+1} f^{(j)}(z)}+\gamma(p-j)\left\{1-\frac{D_{p}^{n} f^{(j)}(z) D_{p}^{n+2} f^{(j)}(z)}{\left[D_{p}^{n+1} f^{(j)}(z)\right]^{2}}\right\}=p(z)+\gamma z p^{\prime}(z),
$$

that is,

$$
p(z)+\gamma z p^{\prime}(z) \prec q(z)+\gamma z q^{\prime}(z) .
$$

Therefore, Theorem 1 now follows by applying Lemma 2.

Putting $q(z)=\frac{1+A z}{1+B z}(-1 \leq B<A \leq 1)$ in Theorem 1 , we obtain the following corollary.

Corollary 1. Let $\gamma \in \mathbb{C}^{*}$ and

$$
\Re\left\{\frac{1-B z}{1+B z}\right\}>\max \left\{0,-\Re\left(\frac{1}{\gamma}\right)\right\} .
$$

If $f \in \mathcal{A}(p)$ satisfy the following subordination condition:

$$
\frac{D_{p}^{n} f^{(j)}(z)}{D_{p}^{n+1} f^{(j)}(z)}+\gamma(p-j)\left\{1-\frac{D_{p}^{n} f^{(j)}(z) D_{p}^{n+2} f^{(j)}(z)}{\left[D_{p}^{n+1} f^{(j)}(z)\right]^{2}}\right\} \prec \frac{1+A z}{1+B z}+\gamma \frac{(A-B) z}{(1+B z)^{2}},
$$


then

$$
\frac{D_{p}^{n} f^{(j)}(z)}{D_{p}^{n+1} f^{(j)}(z)} \prec \frac{1+A z}{1+B z}
$$

and the function $\frac{1+A z}{1+B z}$ is the best dominant.

Taking $p=1$ and $j=0$ in Theorem 1 , we obtain the following subordination result for Sălăgean operator which improves the result of Shanmugam et al. [14, Theorem 5.1] and also obtained by Nechita [11, Corollary 7].

Corollary 2. Let $q(z)$ be univalent in $U$ with $q(0)=1$, and $\gamma \in \mathbb{C}^{*}$. Further assume that (3.1) holds. If $f \in \mathcal{A}(1)$ satisfies the following subordination condition:

$$
\frac{D^{n} f(z)}{D^{n+1} f(z)}+\gamma\left\{1-\frac{D^{n} f(z) D^{n+2} f(z)}{\left[D^{n+1} f(z)\right]^{2}}\right\} \prec q(z)+\gamma z q^{\prime}(z)
$$

then

$$
\frac{D^{n} f(z)}{D^{n+1} f(z)} \prec q(z)
$$

and $q(z)$ is the best dominant.

Remark 1 . Taking $n=j=0$ and $p=1$ in Theorem 1, we obtain the subordination result of Shanmugam et al. [14, Theorem 3.1].

Now, by appealing to Lemma 4 it can be easily prove the following theorem.

Theorem 2. Let $q(z)$ be convex univalent in $U$ with $q(0)=1$. Let $\gamma \in \mathbb{C}$ with $\Re(\bar{\gamma})>0$. If $f \in \mathcal{A}(p)$ such that $\frac{D_{p}^{n} f^{(j)}(z)}{D_{p}^{n+1} f^{(j)}(z)} \in H[q(0), 1] \cap Q$,

$$
\frac{D_{p}^{n} f^{(j)}(z)}{D_{p}^{n+1} f^{(j)}(z)}+\gamma(p-j)\left\{1-\frac{D_{p}^{n} f^{(j)}(z) D_{p}^{n+2} f^{(j)}(z)}{\left[D_{p}^{n+1} f^{(j)}(z)\right]^{2}}\right\}
$$

is univalent in $U$, and the following superordination condition

$$
q(z)+\gamma z q^{\prime}(z) \prec \frac{D_{p}^{n} f^{(j)}(z)}{D_{p}^{n+1} f^{(j)}(z)}+\gamma(p-j)\left\{1-\frac{D_{p}^{n} f^{(j)}(z) D_{p}^{n+2} f^{(j)}(z)}{\left[D_{p}^{n+1} f^{(j)}(z)\right]^{2}}\right\}
$$

holds, then

$$
q(z) \prec \frac{D_{p}^{n} f^{(j)}(z)}{D_{p}^{n+1} f^{(j)}(z)}
$$

and $q(z)$ is the best subordinant.

Taking $q(z)=\frac{1+A z}{1+B z}(-1 \leq B<A \leq 1)$ in Theorem 2, we have the following corollary. 
Corollary 3. Let $\gamma \in \mathbb{C}$ with $\Re(\bar{\gamma})>0$. If $f \in \mathcal{A}(p)$ such that $\frac{D_{p}^{n} f^{(j)}(z)}{D_{p}^{n+1} f^{(j)}(z)} \in$ $H[q(0), 1] \cap Q$,

$$
\frac{D_{p}^{n} f^{(j)}(z)}{D_{p}^{n+1} f^{(j)}(z)}+\gamma(p-j)\left\{1-\frac{D_{p}^{n} f^{(j)}(z) D_{p}^{n+2} f^{(j)}(z)}{\left[D_{p}^{n+1} f^{(j)}(z)\right]^{2}}\right\}
$$

is univalent in $U$, and the following superordination condition

$\frac{1+A z}{1+B z}+\gamma \frac{(A-B) z}{(1+B z)^{2}} \prec \frac{D_{p}^{n} f^{(j)}(z)}{D_{p}^{n+1} f^{(j)}(z)}+\gamma(p-j)\left\{1-\frac{D_{p}^{n} f^{(j)}(z) D_{p}^{n+2} f^{(j)}(z)}{\left[D_{p}^{n+1} f^{(j)}(z)\right]^{2}}\right\}$

holds, then

$$
\frac{1+A z}{1+B z} \prec \frac{D_{p}^{n} f^{(j)}(z)}{D_{p}^{n+1} f^{(j)}(z)}
$$

and $q(z)$ is the best subordinant.

Taking $p=1$ and $j=0$ in Theorem 2, we obtain the following superordination result for Sălăgean operator which improves the result of Shanmugam et al. [14, Theorem 5.2] and also obtained by Nechita [11, Corollary 12].

Corollary 4. Let $q(z)$ be convex univalent in $U$ with $q(0)=1$. Let $\gamma \in \mathbb{C}$ with $\Re(\bar{\gamma})>0$. If $f \in \mathcal{A}(1)$ such that $\frac{D^{n} f(z)}{D^{n+1} f(z)} \in H[q(0), 1] \cap Q$,

$$
\frac{D^{n} f(z)}{D^{n+1} f(z)}+\gamma\left\{1-\frac{D^{n} f(z) \cdot D^{n+2} f(z)}{\left[D^{n+1} f(z)\right]^{2}}\right\}
$$

is univalent in $U$, and the following superordination condition

$$
q(z)+\gamma z q^{\prime}(z) \prec \frac{D^{n} f(z)}{D^{n+1} f(z)}+\gamma\left\{1-\frac{D^{n} f(z) \cdot D^{n+2} f(z)}{\left[D^{n+1} f(z)\right]^{2}}\right\}
$$

holds, then

$$
q(z) \prec \frac{D^{n} f(z)}{D^{n+1} f(z)}
$$

and $q(z)$ is the best subordinant.

Remark 2. Taking $j=n=0$ and $p=1$ in Theorem 2, we obtain the superordination result of Shanmugam et al. [14, Theorem 3.2].

Combining Theorem 1 and Theorem 2, we get the following sandwich theorem for the linear operator $D_{p}^{n} f^{(j)}(z)$.

Theorem 3. Let $q_{1}(z)$ be convex univalent in $U$ with $q_{1}(0)=1, \gamma \in \mathbb{C}$ with $\Re(\bar{\gamma})>0, q_{2}(z)$ be univalent in $U$ with $q_{2}(0)=1$, and satisfies (3.1). If $f \in \mathcal{A}(p)$ such that $\frac{D_{p}^{n} f^{(j)}(z)}{D_{p}^{n+1} f^{(j)}(z)} \in H[q(0), 1] \cap Q$,

$$
\frac{D_{p}^{n} f^{(j)}(z)}{D_{p}^{n+1} f^{(j)}(z)}+\gamma(p-j)\left\{1-\frac{D_{p}^{n} f^{(j)}(z) D_{p}^{n+2} f^{(j)}(z)}{\left[D_{p}^{n+1} f^{(j)}(z)\right]^{2}}\right\}
$$


is univalent in $U$, and

$$
\begin{aligned}
q_{1}(z)+\gamma z q_{1}^{\prime}(z) & \prec \frac{D_{p}^{n} f^{(j)}(z)}{D_{p}^{n+1} f^{(j)}(z)}+\gamma(p-j)\left\{1-\frac{D_{p}^{n} f^{(j)}(z) D_{p}^{n+2} f^{(j)}(z)}{\left[D_{p}^{n+1} f^{(j)}(z)\right]^{2}}\right\} \\
& \prec q_{2}(z)+\gamma z q_{2}^{\prime}(z)
\end{aligned}
$$

holds, then

$$
q_{1}(z) \prec \frac{D_{p}^{n} f^{(j)}(z)}{D_{p}^{n+1} f^{(j)}(z)} \prec q_{2}(z)
$$

and $q_{1}(z)$ and $q_{2}(z)$ are, respectively, the best subordinant and the best dominant.

Taking $q_{i}(z)=\frac{1+A_{i} z}{1+B_{i} z}\left(i=1,2 ;-1 \leq B_{2} \leq B_{1}<A_{1} \leq A_{2} \leq 1\right)$ in Theorem 3 , we obtain the following corollary.

Corollary 5. Let $\gamma \in \mathbb{C}$ with $\Re(\bar{\gamma})>0$. If $f \in \mathcal{A}(p)$ such that $\frac{D_{p}^{n} f^{(j)}(z)}{D_{p}^{n+1} f^{(j)}(z)} \in$ $H[q(0), 1] \cap Q$,

$$
\frac{D_{p}^{n} f^{(j)}(z)}{D_{p}^{n+1} f^{(j)}(z)}+\gamma(p-j)\left\{1-\frac{D_{p}^{n} f^{(j)}(z) D_{p}^{n+2} f^{(j)}(z)}{\left[D_{p}^{n+1} f^{(j)}(z)\right]^{2}}\right\}
$$

is univalent in $U$, and

$$
\begin{aligned}
& \frac{1+A_{1} z}{1+B_{1} z}+\frac{\gamma}{\lambda} \frac{\left(A_{1}-B_{1}\right) z}{\left(1+B_{1} z\right)^{2}} \\
\prec & \frac{D_{p}^{n} f^{(j)}(z)}{D_{p}^{n+1} f^{(j)}(z)}+\gamma(p-j)\left\{1-\frac{D_{p}^{n} f^{(j)}(z) D_{p}^{n+2} f^{(j)}(z)}{\left[D_{p}^{n+1} f^{(j)}(z)\right]^{2}}\right\} \\
\prec & \frac{1+A_{2} z}{1+B_{2} z}+\frac{\gamma}{\lambda} \frac{\left(A_{2}-B_{2}\right) z}{\left(1+B_{2} z\right)^{2}}
\end{aligned}
$$

holds, then

$$
\frac{1+A_{1} z}{1+B_{1} z} \prec \frac{D_{p}^{n} f^{(j)}(z)}{D_{p}^{n+1} f^{(j)}(z)} \prec \frac{1+A_{2} z}{1+B_{2} z}
$$

and $\frac{1+A_{1} z}{1+B_{1} z}$ and $\frac{1+A_{2} z}{1+B_{2} z}$ are, respectively, the best subordinant and the best dominant.

Taking $p=1$ and $j=0$ in Theorem 3, we obtain the following sandwich result for Sălăgean operator which improves the result of Shanmugam et al. [14, Theorem 5.3].

Corollary 6. Let $q_{1}(z)$ be convex univalent in $U$ with $q_{1}(0)=1, \gamma \in \mathbb{C}$ with $\Re(\bar{\gamma})>0, q_{2}(z)$ be univalent in $U$ with $q_{2}(0)=1$, and satisfies (3.1). If $f \in \mathcal{A}(1)$ such that $\frac{D^{n} f(z)}{D^{n+1} f(z)} \in H[q(0), 1] \cap Q$,

$$
\frac{D^{n} f(z)}{D^{n+1} f(z)}+\gamma\left\{1-\frac{D^{n} f(z) \cdot D^{n+2} f(z)}{\left[D^{n+1} f(z)\right]^{2}}\right\}
$$


is univalent in $U$, and

$q_{1}(z)+\gamma z q_{1}^{\prime}(z) \prec \frac{D^{n} f(z)}{D^{n+1} f(z)}+\gamma\left\{1-\frac{D^{n} f(z) \cdot D^{n+2} f(z)}{\left[D^{n+1} f(z)\right]^{2}}\right\} \prec q_{2}(z)+\gamma z q_{2}^{\prime}(z)$

holds, then

$$
q_{1}(z) \prec \frac{D^{n} f(z)}{D^{n+1} f(z)} \prec q_{2}(z)
$$

and $q_{1}(z)$ and $q_{2}(z)$ are, respectively, the best subordinant and the best dominant.

Remark 3. Taking $n=j=0$ and $p=1$ in Theorem 3, we obtain the sandwich result of Shanmugam et al. [14, Corollary 3.3].

\section{References}

[1] R. M. Ali, V. Ravichandran, M. H. Khan, and K. G. Subramanian, Differential sandwich theorems for certain analytic functions, Far East J. Math. Sci. (FJMS) 15 (2004), no. 1, 87-94.

[2] M. K. Aouf, Generalization of certain subclasses of multivalent functions with negative coefficients defined by using a differential operator, Math. Comput. Modelling 50 (2009), no. 9-10, 1367-1378.

[3] _ On certain multivalent functions with negative coefficients defined by using a differential operator, Indian J. Math. 51 (2009), no. 2, 433-451.

[4] M. K. Aouf and A. O. Mostafa, On a subclass of $n$-p-valent prestarlike functions, Comput. Math. Appl. 55 (2008), no. 4, 851-861.

[5] T. Bulboacă, Classes of first-order differential superordinations, Demonstratio Math. 35 (2002), no. 2, 287-292.

[6] - Differential Subordinations and Superordinations, Recent Results, House of Science Book Publ. Cluj-Napoca, 2005.

[7] B. C. Carlson and D. B. Shaffer, Starlike and prestarlike hypergeometric functions, SIAM J. Math. Anal. 15 (1984), no. 4, 737-745.

[8] M. Kamali and H. Orhan, On a subclass of certain starlike functions with negative coefficients, Bull. Korean Math. Soc. 41 (2004), no. 1, 53-71.

[9] S. S. Miller and P. T. Mocanu, Differential Subordination, Monographs and Textbooks in Pure and Applied Mathematics, 225. Marcel Dekker, Inc., New York, 2000.

[10] Subordinants of differential superordinations, Complex Var. Theory Appl. 48 (2003), no. 10, 815-826.

[11] V. O. Nechita, Differential subordinations and superordinations for analytic functions defined by the generalized Sălăgean derivative, Acta Univ. Apulensis Math. Inform. No. 16 (2008), 143-156.

[12] S. Ruscheweyh, New criteria for univalent functions, Proc. Amer. Math. Soc. 49 (1975), 109-115.

[13] G. S. Sălăgean, Subclasses of univalent functions, Complex analysis - fifth RomanianFinnish seminar, Part 1 (Bucharest, 1981), 362-372, Lecture Notes in Math., 1013, Springer, Berlin, 1983.

[14] T. N. Shanmugam, V. Ravichandran, and S. Sivasubramanian, Differantial sandwich theorems for some subclasses of analytic functions, J. Austr.Math. Anal. Appl. 3 (2006), no. 1, Art. 8, 1-11.

[15] N. Tuneski, On certain sufficient conditions for starlikeness, Int. J. Math. Math. Sci. 23 (2000), no. 8, 521-527. 
Mohamed K. Aouf

Department of Mathematics

FACUlty of SCIENCE

MANSOURA UnIVERSITY

MAnsoura 35516, EGyPT

E-mail address: mkaouf127@yahoo.com

TAmer M. SEOUdy

Department of Mathematics

FACUlty OF SCIENCE

FAYOUM UNIVERSITY

FAYOUM 63514, EGYPT

E-mail address: tmseoudy@gmail.com 